\title{
CONTROL ECOGRÁFICO POST INSERCIÓN DE DISPOSITIVO INTRAUTERINO
}

\author{
Daniel Veloso M. ${ }^{1}$, Germán Lobos A. ${ }^{a}$, Norma Aliste S. ${ }^{b}$, Carla Rojas G. ${ }^{b}$, \\ Pilar García M. ${ }^{b}$, Pascal Patrick Matzler. ${ }^{c}$ \\ 1 CESFAM Carlos Trupp y Facultad de Ciencias de la Salud, Universidad Católica del Maule, Talca, Chile.
}

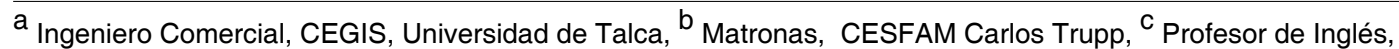
Instituto de Estudios Generales, Universidad Católica del Maule, Talca, Chile.

\section{RESUMEN}

Introducción: Los dispositivos intrauterinos (DIU) son métodos de anticoncepción reversible, ampliamente usados en el mundo. Se estima que 120 millones de mujeres usan un DIU en el mundo como método anticonceptivo. Objetivo: Evaluar los factores de riesgo que puedan relacionarse con una incorrecta inserción del DIU. Método: 93 pacientes de un Centro de Salud Familiar de la ciudad de Talca, Chile. Utilizando tablas de contingencia se calcularon diferentes medidas de asociación y se compararon los casos de DIU mal posicionado con los casos con uno o más factores de riesgo identificados. Resultados: Se encontró una incidencia de DIU incorrectamente posicionado de un 15,8\%, de los cuales hubo un 2,1\% de perforación uterina, 2,1\% para DIU incrustado y $11,8 \%$ para DIU descendido. Se identificaron como factores de riesgo de DIU incorrectamente posicionado la cicatriz de cesárea (RR: 2,31), inserción dificultosa (RR: 3,31) y opinión de un resultado dudoso (RR: 3,64), con una sensibilidad de 73,3\%. Conclusiones: La ecotomografía transvaginal es una herramienta crucial para la confirmación de una correcta inserción del DIU y debería ser un examen de rutina ante la presencia de cualquiera de los factores de riesgo identificados.

\section{PALABRAS CLAVE: Dispositivo intrauterino, factores de riesgo, ecotomografía transvaginal}

\section{SUMMARY}

Background: Intrauterine devices (IUDs) are reversible birth control methods that are widely used throughout the world. It is estimated that 120 million women use an IUD in the world as a contraceptive. Aim: To assess the risk factors that may relate to incorrect insertion of the IUD. Methods: The sample included 93 patients of a Family Health Center, Talca, Chile. Using contingency tables, various measures of association were calculated, and the cases of incorrectly positioned IUD were compared with the cases where one or more risk factors were identified. Results: We found an incidence of $15.8 \%$ of incorrectly positioned IUD, of which $2.1 \%$ presented with uterine perforation, $2.1 \%$ with embedment of the IUD and $11.8 \%$ with a descended IUD. We identified the following risk factors for incorrectly positioned IUD: cesarean section (RR: 2.31), difficulty on insertion (RR: 3.31) and practitioner's opinion of an uncertain result (RR: 3.64), with a sensitivity of $73.3 \%$. Conclusions: Transvaginal ultrasound is a crucial tool for confirmation of proper insertion of an IUD and should be considered a routine examination when any of the identified risk factors are present.

\section{KEYWORD: Intrauterine device, risk factors, transvaginal ultrasonography}




\section{INTRODUCCIÓN}

Los dispositivos intrauterinos (DIU) son métodos de anticoncepción (1) usados en una proporción muy variable en distintos países (2). En Chile es usado por alrededor del $55 \%$ de las mujeres adscritas al sistema público de salud (3).

EI DIU ofrece la mejor relación costo-efectividad (4), por lo que debería ser de primera línea entre las opciones de métodos de anticoncepción (MAC) disponibles (5). El riesgo de enfermedad inflamatoria pélvica (EIP) es de 1-2 eventos por cada 1.000 mujeres/año, similar al riesgo de no usuarias del DIU (6). EI DIU no aumenta el riesgo de infertilidad tubaria, embarazo ectópico y EIP (7). Una investigación reciente reportó una tasa de pérdida reproductiva de embarazos con DIU del $40 \%$ (8).

EI DIU con cobre más usado en Chile es la T-Cu 380A, aprobado por FDA (9). El mecanismo de acción anticonceptiva de los DIU con cobre es diverso (10), incluso podría inhibir el transporte espermático (11).

El sangrado excesivo y el dolor son efectos adversos del DIU (12), lo cual ocurre en 1/1.000 inserciones, pudiendo superar una frecuencia de $1 / 100$ inserciones (13), dependiendo de variables como experiencia del operador, tamaño y posición del útero, presencia de malformaciones uterinas y periodo de posparto o post aborto (14).

Las guías de Lippes (15) fueron de gran utilidad para el control de la posición correcta del DIU junto con la radiografía. Actualmente la ecografía ginecológica transvaginal es el patrón de oro del control del DIU. Diversos autores han utilizado la distancia entre el extremo superior del dispositivo y el fondo del útero, el fondo del endometrio y desde el endometrio-miometrio, como indicadores de la posición correcta del DIU $(16,17)$.

El presente estudio tiene como objetivo principal evaluar mediante la ultrasonografía los factores de riesgo que puedan relacionarse con una incorrecta inserción del DIU.

\section{PACIENTES Y MÉTODOS}

En una unidad de atención primaria en salud de la ciudad de Talca, Chile, se realizó un estudio de cohorte de mujeres que cumplen con los criterios de selección de la OMS y las Normas Nacionales de Regulación de la Fertilidad del Ministerio de Salud (MINSAL) (18) para el uso de DIU, a las que se insertó una T-Cu 380A. Participaron un total de tres matronas autorizadas y capacitadas para realizar el procedimiento de inserción de DIU y un médico especialista en ginecología y obstetricia. Una matrona fue capacitada y entrenada en el control ecográfico, que realizó las evaluaciones con un ecógrafo con transductor vaginal el mismo día de la inserción. Se aplicó un instrumento para evaluar factores de riesgo de mala inserción con variables clínicas (Tabla I) y variables ecográficas (Tabla II). Consideramos una correcta ubicación del DIU (in situ) cuando su rama longitudinal se encuentra completamente dentro de la cavidad endometrial, tomando como límite proximal el fondo del endometrio y como límite distal, el endocervix, definido como el punto de transición entre la capa endometrial y la linea endocervical. Se consideró como DIU mal posicionado (heterotópico) a aquel que no cumple con esta condición, siendo divididos en DIU descendido (la rama longitudinal se encuentra ocupando el endocervix en una longitud mayor a la distancia del extremo proximal al fondo del endometrio), incrustado (cualquier parte del dispositivo atraviesa el endometrio) y ectópico o extrauterino (el DIU ha salido fuera de los límites de la serosa uterina).

Con el objeto de contrastar la independencia o asociación entre variables categóricas nominales, se realizó un análisis descriptivo basado en tablas de contingencia, considerando como variable de referencia la posición del DIU por ecografía $(0=$ in situ, 1=heterotópico) y como variables de contraste diversos factores de riesgo de variables clínicas y ecográficas: número de partos vaginales (NPV), número de partos por cesárea (NPC), número de inserción (NIN), posición del útero por clínica (PUC),

\section{Tabla I}

\section{VARIABLES CLÍNICAS PARA EVALUAR FACTORES DE RIESGO DE MALA INSERCIÓN}

\begin{tabular}{|c|c|}
\hline Variable & Escala \\
\hline Número de partos por cesárea & $\mathrm{n}$ \\
\hline Número de partos vaginales & $\mathrm{n}$ \\
\hline \multirow[t]{3}{*}{$\begin{array}{l}\text { Número de inserciones de DIU } \\
\text { previas }\end{array}$} & $\mathrm{n}$ \\
\hline & Anteversión \\
\hline & $\begin{array}{l}\text { Retroversión de } \\
\text { primer grado }\end{array}$ \\
\hline \multirow[t]{2}{*}{ Posición del útero por clínica } & $\begin{array}{l}\text { Retroversión de } \\
\text { segundo grado }\end{array}$ \\
\hline & $\begin{array}{l}\text { Retroversión de } \\
\text { tercer grado }\end{array}$ \\
\hline Histerometría por clínica & $\mathrm{n}(\mathrm{cm})$ \\
\hline Opinión del resultado & $\begin{array}{l}\text { In situ } \\
\text { Con dudas }\end{array}$ \\
\hline Grado de dificultad & $\begin{array}{l}\text { Fácil } \\
\text { Difícil } \\
\text { Muy difícil }\end{array}$ \\
\hline
\end{tabular}

DIU: dispositivo intrauterino. 
histerometría por histerómetro $(\mathrm{HPH})$, opinión del resultado de la inserción (ORI), grado de dificultad de la inserción (GDI), posición del útero por ecografía (PUE), histerometría por ecografía (HPE). La definición de variables se presenta en la Tabla III.

Para el análisis estadístico, los datos fueron recopilados en una planilla electrónica y analizada en el programa SPSS 15. Se organizaron en tablas de doble entrada (tablas de contingencia), donde cada entrada representa un criterio de clasificación

\section{Tabla II}

\section{VARIABLES ECOGRÁFICAS PARA EVALUAR FACTORES DE RIESGO DE MALA INSERCIÓN}

\begin{tabular}{|c|c|c|}
\hline Variable & \multicolumn{2}{|l|}{ Escala } \\
\hline \multicolumn{3}{|l|}{ Histerometría por } \\
\hline ecografía & \multicolumn{2}{|l|}{$\mathrm{n}(\mathrm{cm})$} \\
\hline & \multicolumn{2}{|c|}{$\begin{array}{l}\text { Anteversión } \\
\text { Retroversión de primer } \\
\text { grado }\end{array}$} \\
\hline $\begin{array}{l}\text { Posición del útero por } \\
\text { ecografía }\end{array}$ & \multicolumn{2}{|c|}{$\begin{array}{l}\text { Retroversión de segundo } \\
\text { grado } \\
\text { Retroversión de tercer grado }\end{array}$} \\
\hline Dispositivo intrauterino & $\begin{array}{l}\text { In situ } \\
\text { Heterotópico }\end{array}$ & $\begin{array}{l}\text { Descendido } \\
\text { Incrustado } \\
\text { Ectópico }\end{array}$ \\
\hline
\end{tabular}

(o variable categórica). De esta forma la frecuencia (número de casos) aparece en casillas con información sobre la relación existente entre ambos criterios. En la Tabla IV se presentan las tablas de contingencia donde en todos los casos la variable de fila es la posición del DIU por ecografía (PDIUE).

Para medir independencia o asociación entre las variables y la intensidad de la asociación se utilizó Chi-cuadrado de Pearson $\left(x^{2}\right)$ (19). Se analizaron las variables con estimación de Riesgo Relativo, con un intervalo de confianza del $95 \%$ y un nivel de significancia del $5 \%$.

\section{RESULTADOS}

Se estudiaron un total de 93 mujeres a las que se les insertó un DIU T-Cu 380A entre marzo de 2008 y agosto de 2009, cuyo perfil epidemiológico se describe en la Tabla V. La incidencia de DIU mal posicionado inmediatamente después de la inserción a la ecografía transvaginal fue de un 15,8\%. De ellos, la incidencia de perforación y de incrustación del DIU en el miometrio fue del 2,1\%. Los dos casos de perforación fueron asintomáticas durante el procedimiento, los que se reportaron como dificultosos y con dudas de su resultado: uno de ellos quedó en el espacio vesicouterino, en relación a la cicatriz de cesárea y el otro quedó en el fondo de

Tabla III

DEFINICIÓN DE LA VARIABLE DEPENDIENTE Y LAS VARIABLES INDEPENDIENTES PARA ANÁLISIS DE CONTRASTE

\begin{tabular}{|c|c|}
\hline Variables & Descripción \\
\hline PDIUE & Posición dispositivo intrauterino por ecografía: «0» significa “in situ”, «1» significa "heterotópico" \\
\hline NPV & Número de partos vaginales: «0» indica sin partos vaginales, « $1 »$ indica uno o más partos vaginales \\
\hline NPC & $\begin{array}{l}\text { Número de partos por cesárea : «0» indica sin partos por cesárea, «1» indica uno, «2» indica dos, } \\
\text { «3» indica tres o más partos por cesárea }\end{array}$ \\
\hline NIN & Número de inserción: «0» indica primera inserción, «1» indica segunda inserción o más \\
\hline PUC & $\begin{array}{l}\text { Posición del útero por clínica: «0» indica si la posición del útero es en anteversión, «1» indica retro- } \\
\text { versión leve a moderada, « } 2 » \text { indica retroversión severa }\end{array}$ \\
\hline $\mathrm{HPH}$ & $\begin{array}{l}\text { Histerometría por histerómetro: «0» si el resultado es } 6 \text { a } 7 \mathrm{~cm} \text {, « } 1 \text { » si es entre } 7 \text { y } 8 \mathrm{~cm} \text {, «2» si es } \\
\text { entre } 8 \text { y } 9 \mathrm{~cm} \text {, « } 3 \text { » si es } 9 \text { o más cm }\end{array}$ \\
\hline ORI & $\begin{array}{l}\text { Opinión del resultado de la inserción: «0» si la opinión del resultado es "in situ”, «1» si la opinión del } \\
\text { resultado es "con dudas", « } 2 \text { » si la opinión del resultado es "muy dudoso" }\end{array}$ \\
\hline GDI & $\begin{array}{l}\text { Grado de dificultad de la inserción: «0» si la opinión del resultado es "fácil”, «1» si la opinión del } \\
\text { resultado es "difícil”, «2» si la opinión del resultado es "muy difícil” }\end{array}$ \\
\hline PUE & $\begin{array}{l}\text { Posición del útero por ecografía: «0» indica si la posición del útero es en anteversión, «1» si la } \\
\text { posición del útero es en retroversión de I a II grado, «2» si la posición del útero es en retroversión } \\
\text { de III grado }\end{array}$ \\
\hline HPE & $\begin{array}{l}\text { Histerometría por ecografía: «0» si el resultado es } 6 \text { a } 7 \mathrm{~cm} \text {, «1» si es entre } 7 \text { y } 8 \mathrm{~cm} \text {, «2» si es entre } \\
8 \text { y } 9 \mathrm{~cm} \text {, «3» si es } 9 \text { o más cm }\end{array}$ \\
\hline
\end{tabular}


saco posterior. Ambos casos se solucionaron posteriormente por laparoscopía y por culdotomía respectivamente. Los dos DIU incrustados en el miometrio fueron retirados por tracción de las guías sin complicaciones. Un 11,8\% se encontraba descendido parcialmente, ocupando parte del endocérvix. De ellos, 2 fueron extraídos por encontrarse con más de la mitad de su rama en el canal cervical, en el resto, el DIU se dejó in situ (9 casos) (Tabla V).

Se observó una intensidad de asociación estadísticamente significativa entre la posición del DIU por ecografía (variable de referencia) y opinión del resultado de la inserción (ORI) y grado de dificultad de la inserción (GDI), ambas al valor $p<0,01$.
También se observó una intensidad de asociación estadísticamente significativa entre la variable de referencia y número de partos por cesárea (NPC), al valor $\mathrm{p}<0,05$ (Tabla VI y Tabla VII).

Al agrupar las variables con uno o más factores clínicos de asociación estadísticamente significativas encontradas en la Tabla VI (GDI, ORI, NPC), considerados como de riesgo para los casos de PDIUE en una tabla de doble entrada, se encontró que ante una prueba positiva para factores de riesgo, existe un $73,3 \%$ de probabilidad que se detecten a las mujeres con DIU "heterotópico" y ante una prueba negativa, existe un $92,6 \%$ de que el DIU esté "in situ" (Tabla VII).

Tabla IV

TABLAS DE CONTINGENCIA ENTRE LA POSICIÓN DEL DIU POR ECOGRAFÍA Y FACTORES DE RIESGO DE VARIABLES CLÍNICAS Y ECOGRÁFICAS

\begin{tabular}{|c|c|c|c|c|c|c|}
\hline & & $N P V=0$ & $N P V=1$ & & & Total \\
\hline \multirow[t]{2}{*}{ PDIUE } & 0 & 15 & 63 & & & 78 \\
\hline & 1 & 6 & 9 & & & 15 \\
\hline \multirow[t]{2}{*}{ Total } & & 21 & 72 & & & 93 \\
\hline & & $\mathrm{NPC}=0$ & $\mathrm{NPC}=1$ & $\mathrm{NPC}=2$ & $\mathrm{NPC}=3$ & Total \\
\hline \multirow[t]{2}{*}{ PDIUE } & 0 & 60 & 11 & 5 & 2 & 78 \\
\hline & 1 & 7 & 6 & 1 & 1 & 15 \\
\hline Total & & $\begin{array}{c}67 \\
\mathrm{NIN}=0\end{array}$ & $\begin{array}{c}17 \\
\mathrm{NIN}=1\end{array}$ & 6 & 3 & $\begin{array}{c}93 \\
\text { Total }\end{array}$ \\
\hline \multirow[t]{2}{*}{ PDIUE } & 0 & 45 & 33 & & & 78 \\
\hline & 1 & 7 & 8 & & & 15 \\
\hline \multirow[t]{2}{*}{ Total } & & 52 & 41 & & & 93 \\
\hline & & $P U C=0$ & $P U C=1$ & $P U C=2$ & & Total \\
\hline \multirow[t]{2}{*}{ PDIUE } & 0 & 64 & 12 & 2 & & 78 \\
\hline & 1 & 13 & 2 & 0 & & 15 \\
\hline \multirow[t]{2}{*}{ Total } & & 77 & 14 & 2 & & 93 \\
\hline & & $\mathrm{HPH}=0$ & $\mathrm{HPH}=1$ & $\mathrm{HPH}=2$ & $\mathrm{HPH}=3$ & Total \\
\hline \multirow[t]{2}{*}{ PDIUE } & 0 & 19 & 34 & 18 & 6 & 78 \\
\hline & 1 & 4 & 7 & 3 & 1 & 15 \\
\hline \multirow[t]{2}{*}{ Total } & & 23 & 41 & 21 & 7 & 92 \\
\hline & & $\mathrm{ORI}=0$ & $\mathrm{ORI}=1$ & $\mathrm{ORI}=2$ & & Total \\
\hline \multirow[t]{2}{*}{ PDIUE } & 0 & 68 & 8 & 2 & & 78 \\
\hline & 1 & 8 & 5 & 2 & & 15 \\
\hline \multirow[t]{2}{*}{ Total } & & 76 & 13 & 4 & & 93 \\
\hline & & $\mathrm{GDI}=0$ & $\mathrm{GDI}=1$ & $\mathrm{GDI}=2$ & & Total \\
\hline \multirow[t]{2}{*}{ PDIUE } & 0 & 67 & 11 & 0 & & 78 \\
\hline & 1 & 8 & 5 & 2 & & 15 \\
\hline \multirow[t]{2}{*}{ Total } & & 75 & 16 & 2 & & 93 \\
\hline & & $\mathrm{PUE}=0$ & $P U E=1$ & PUE=2 & & Total \\
\hline \multirow[t]{2}{*}{ PDIUE } & 0 & 54 & 22 & 2 & & 78 \\
\hline & 1 & 9 & 5 & 1 & & 15 \\
\hline \multirow[t]{2}{*}{ Total } & & 63 & 27 & 3 & & 93 \\
\hline & & $\mathrm{HPE}=0$ & $\mathrm{HPE}=1$ & HPE=2 & HPE=3 & Total \\
\hline \multirow[t]{2}{*}{ PDIUE } & 0 & 6 & 13 & 34 & 24 & 77 \\
\hline & 1 & 4 & 1 & 4 & 6 & 15 \\
\hline Total & & 10 & 14 & 38 & 30 & 92 \\
\hline
\end{tabular}

DIU: dispositivo intrauterino. PDIUE: posición del DIU por ecografía. 
Tabla V

PERFIL EPIDEMIOLÓGICO DE LA MUESTRA

\begin{tabular}{|c|c|}
\hline Variables & $\mathrm{n}=93$ \\
\hline Edad [mediana ( \pm DS)] (años) & $27,6(8,2)$ \\
\hline Paridad [promedio ( $\pm \mathrm{DS})]$ & $1,8(1,0)$ \\
\hline Cesáreas previas [promedio ( \pm DS)] & $0,4(0,8)$ \\
\hline Partos vaginales [promedio ( \pm DS)] & $1,7(0,8)$ \\
\hline \multicolumn{2}{|l|}{ Inserciones [n (\%)] } \\
\hline Primera inserción & $52(56 \%)$ \\
\hline Segunda o más inserción & $41(44 \%)$ \\
\hline \multicolumn{2}{|l|}{ Posición del DIU [n (\%)] } \\
\hline DIU descendido & $11(11,8 \%)$ \\
\hline DIU incrustado & $2(2,1 \%)$ \\
\hline DIU extrauterino & $2(2,1 \%)$ \\
\hline
\end{tabular}

DS: desvío estándar.

Tabla VI

FACTORES DE RIESGO DE POSICIÓN DIU POR ECOGRAFÍA HETEROTÓPICO

\begin{tabular}{|c|c|c|c|c|}
\hline Variable & $x^{2}$ & $\begin{array}{l}\text { Riesgo } \\
\text { relativo }\end{array}$ & IC 95\% & Valor $p$ \\
\hline NPV & 3,1 & 2,08 & $(0,96-4,49)$ & 0,096 \\
\hline $\mathrm{NPC}^{\circ \circ}$ & 5,7 & 2,31 & $(1,24-4,31)$ & $0,026^{*}$ \\
\hline NIN & 0,62 & 0,81 & $(0,46-1,44)$ & 0,27 \\
\hline $\mathrm{PUC}^{\circ \circ}$ & 0,19 & 0,74 & $(0,19-2,94)$ & 1,0 \\
\hline $\mathrm{HPH}^{\mathrm{oo}, \mathrm{a}}$ & 0,03 & 1,08 & $(0,43-2,73)$ & 1,0 \\
\hline $\mathrm{OR} 1^{\circ \mathrm{O}}$ & 9,65 & 3,64 & $(1,65-8,04)$ & $0,005^{\star}$ \\
\hline $\mathrm{GDI}^{\circ \circ}$ & 8,55 & 3,31 & $(1,53-7,15)$ & $0,008^{*}$ \\
\hline PUE $^{\circ o}$ & 0,49 & 1,30 & $(0,64-2,63)$ & 0,55 \\
\hline $\mathrm{HPE}^{\mathrm{oo}, \mathrm{a}}$ & 4,62 & 3,42 & $(1,10-10,68)$ & $0,05^{\star}$ \\
\hline
\end{tabular}

* Estadísticamente significativo: $p \leq 0,05$. ${ }^{\circ}$ Para $0=0$ y 1 o más $=1 .{ }^{a}$ Mayor riesgo a mayor tamaño del útero

Tabla VII

CASOS VS FACTORES DE RIESGO

\begin{tabular}{cccc}
\hline & & \multicolumn{2}{c}{ Factores de riesgo } \\
& Score 0 & Score $\geq 1$ \\
\hline DIU & Heterotópico & 11 & 4 \\
& In situ & 28 & 50 \\
RR: $2,04(1,31-3,13)$ & Sensibilidad: $73,3 \%$ \\
$x^{2}: 7,24 ; \mathrm{p}=0,007$ & VP negativo: $92,6 \%$ \\
\hline
\end{tabular}

\section{DISCUSIÓN}

Se encontró un incidencia alta de perforación $(2,1 \%)$, muy superior a la reportada en la literatura internacional que varía en 0,8 a 1,6 por 1000 inserciones (13-14). Los autores piensan que esto se debe a que el número de operadores que participaron en el estudio es reducido y puede ser un grupo no representativo. Respecto a los DIU descendidos $(11,8 \%)$, estos corresponden a aquellos que ocupan parcial o totalmente el endocérvix. Este resultado no puede ser comparado con los estudios existentes, ya que los parámetros para definir el concepto "DIU descendido" son diferentes entre los diferentes trabajos analizados. En el trabajo de Inal y cols (8) utilizaron un grupo control de 300 mujeres con una incidencia del $11 \%$ de DIU CuT380A descendido, siendo más frecuente en mujeres menores de 21 años y con sólo 1 parto previo. Respecto a la posibilidad de "acomodación" o movilidad del DIU durante el ciclo menstrual, hay información controvertida al respecto. Faúndes y cols (17), informan que el DIU en forma de T acomoda su posición dentro del útero los primeros 3 meses después de la inserción y que la evaluación por ecografía no es un buen predictor. Se atribuye este fenómeno a la contractilidad uterina (14). Por otra parte, Salamanca y cols (11), en un seguimiento de 25 mujeres con contractilidad uterina subendometrial-miometrial demostrada por ecografía antes de insertar un DIU TCu-340, evidenció que el $100 \%$ de ellas pierde esta característica al año de seguimiento, con una pérdida completa de la contractilidad retrógrada (del cérvix al fondo) desde el primer mes de seguimiento. Estos hallazgos hacen pensar, aunque no con certeza, que un DIU con cobre con su vástago que ocupa parte del endocérvix o que se encuentra muy alejado del fondo, puede permanecer descendido a lo largo del tiempo.

Múltiples factores de riesgo de perforación uterina se describen en la literatura, muchos de ellos sin un fundamento claro. En Turquía, un estudio de Caliskan y cols (14), con una muestra de 8343 mujeres usuarias de TCu380A, analizaron múltiples factores de riesgo tales como paridad, abortos previos, cesáreas, tiempo de inserción post parto y experiencia del operador. Sólo pudo encontrar como factor de riesgo la inserción antes de 6 meses después del parto. En nuestro estudio, a diferencia del trabajo de Caliskan, encontramos que la cicatriz por cesárea previa es un factor de riesgo de DIU "heterotópico" [RR: 2,31; IC95\% 1,24 - 4,31; p=0,026].

No se encontró en la literatura información sobre la opinión del operador respecto de la dificultad del procedimiento o la sensación del resultado es- 
perado. Solamente hay informes de recomendaciones sobre la técnica y la necesaria capacitación del personal de salud $(2,9,10,18)$, sin embargo, en ninguno de estos estudios consideraron como factores de riesgo la opinión del operador sobre el procedimiento de inserción.

En nuestro estudio encontramos que los factores de riesgo más importantes para DIU "heterotópico" son dependientes de la opinión del operador. El grado de dificultad [RR 3,31; IC95\% 1,53 - 7,15; $p=0,008$ ] y el grado de satisfacción [RR 3,64; IC95\% $1,65-8,04 ; p=0,005]$ referidos por el operador sobre el resultado del procedimiento de inserción, así como el antecedente de cicatriz de cesárea forman una tríada de riesgo significativo, con una sensibilidad del $73,3 \%$ y un valor predictivo negativo del $92,6 \%$, lo que hace que la identificación de estas variables sea de gran importancia.

No incluimos como posibles variables, la inserción inmediatamente post parto o la inserción antes de los 3 meses de puerperio ya que esta práctica es excepcional en nuestra unidad de trabajo.

\section{CONCLUSIONES}

Debido a la alta incidencia de DIU heterotópico encontrada por nuestro grupo $(15,8 \%)$, la ecografía es para nosotros una herramienta muy importante en el control post inserción del DIU y debería realizarse de rutina. La identificación de uno o más factores de riesgo para DIU incorrectamente inserto, tales como cicatriz de cesárea previa, procedimiento de inserción dificultoso o dudas respecto del resultado del procedimiento, hacen obligatorio un control ecográfico inmediatamente después de la inserción. Es necesario un estudio multicéntrico que permita responder si los factores de riesgo identificados por nuestro grupo se repiten en el resto de la red asistencial.

AGRADECIMIENTOS: Los autores agradecen el apoyo del Centro de Salud Familiar (CESFAM) Carlos Trupp de Talca y del Centro de Estudios en Gestión de Instituciones de Salud (CEGIS) de la Universidad de Talca.

\section{BIBLIOGRAFÍA}

1. WHO. 2006. Maternal Mortality Fact Sheet. World Health Organization, Division of Family Health. Geneva, Italy.

2. d'Arcangues $\mathrm{C}$. Worldwide use of intrauterine device for contraception. Contraception 2007;65:389-95.

3. MINSAL. Estadísticas Vitales. Ministerio de Salud de
Chile. Santiago, Chile: MINSAL; 2005, 83-92 pgs.

4. Association of Reproductive Health Professionals. Non hormonal contraceptive methods: a quick reference guide for clinicians. Washington, DC: ARHP; 2006.

5. American College of Obstetricians and Gynecologists. Birth control options for women - intrauterine devices (IUDs). Washington, DC: ACOG; 2007.

6. Stubbs E, Schamp A. The evidence is in. Why are IUDs still out? Family physicians' perceptions of risk and indications. Can Fam Physician 2008;54:560-6.

7. Gold M, Johnson L. Intrauterine devices and adolescents. Curr Opin Obstet Gynecol 2008;20:464-9.

8. Inal MM, Ertopcu K, Ozelmas I. The evaluation of 318 intrauterine pregnancy cases with an intrauterine device. Eur J Contracept Reprod Health Care 2005;10:266-71.

9. Evans AT. Manual of Obstetrics. Seventh Edition. Philadelphia, PA. Lippincott Williams \& Wilkins, 2000;91-9.

10. Alvares $F$, Brache V, Fernández $E$. New insights on the mode of action of intrauterine contraceptive devices in women. Fertil Steril 1988;49:768-73.

11. Salamanca A, Carrillo $M$, Beltrán E, Clavero $P$. Transvaginal sonographic evaluation of subendometrial-myometrial contractility in women using a copper-releasing intrauterine device. Contraception 2008;77:444-6.

12. Fantasia $\mathrm{H}$. Options for intrauterine contraception. $\mathrm{J}$ Obs Gyn Neonat Nurs 2008;37:375-83.

13. Anderson K, Ride-Blomqvist E, Lindell K, Odlind V, Milson I. Perforation with intrauterine devides. Report from a Swedish survey. Contraception 1998;57:251-5.

14. Caliskan E, Oztürk N, Dilbaz BO, Dilbaz S. Analysis of risk factors associated with uterine perforation by intrauterine devices. Eur J Contracept Reprod Health Care 2003;8:150-5.

15. Lippes J. Development of a plastic loop and instructions for the insertion of an intrauterine loop. Proceedings of the First International Conference on Intrauterine Contraception. Excerpta Medica Foundation 1962, Intl Congress Series 54: 69-145.

16. Kratochwil A. Utrachall diagnostik in der gynäkologies. Gynäkologie 1976;9:166-80.

17. Faundes D, Perdigao A, Faundes A, Bahamondes L, Petta CA. T-shaped IUDs accommodate in their position during the first 3 months after insertion. Contraception 2000;62:165-8.

18. MINSAL. Normas Nacionales sobre Regulación de la Fertilidad. Ministerio de Salud de Chile. Santiago, Chile: MINSAL; 2007.

19. Cerda J, Villarroel L. Interpretación del test de chicuadrado $\left(\mathrm{X}^{2}\right)$ en investigación pediátrica. Rev Chil Pediatr 2007;78:414-7.

20. Pearson K. On a criterion that a given system of deviations from the probable in the case of correlated system of variables is Duch that it can be reasonably supposed to have arisen from random sampling. Philosophical Magazine 1900;50:157-75. 